\title{
Abstract \\ De novo papillary carcinoma in a thyroglossal duct cyst
}

Perera K A A J', Gunawardena S A ${ }^{1,}$ Rodrigo $S^{1}$

${ }^{1}$ Sri Jayawardenapura General Hospital, Sri Lanka

Key words: papillary carcinoma, thyroglossal duct cyst

\section{Introduction}

Thyroglossal duct cysts (TGDC) account for $70 \%$ of congenital neck masses. Majority $(70 \%)$ is diagnosed during childhood and only $7 \%$ in adulthood. Only $1 \%$ of thyroid carcinomas evolve from a TGDC. It's essential to differentiate primary papillary carcinoma in a TGDC from a metastatic papillary carcinoma of thyroid.

Objective

A 23 year old girl presented with a painless lump over the anterior neck for one month. Examination revealed a non-tender firm midline neck lump just above the hyoid bone with no palpable thyroid nodules or cervical lymphadenopathy. Ultrasound revealed a suprahyoid TGDC and a sub-centimeter solitary thyroid nodule (STN) in right lobe with no cervical lymphadenopathy. FNAC of TGDC showed a benign cystic neoplasm. Ultrasound guided FNAC of the STN showed benign cells only. Thyroid functions were normal. A Sistrunk operation was performed. Histology revealed a papillary carcinoma (with CTF1 nuclear and CK19 positivity in cells lining the papillae).

A Total Thyroidectomy was performed after an oncological consultation to exclude a possible primary focus in the STN and to facilitate adjuvant treatment. Histology of the STN was a papillomatous focus likely to be hyperplastic (CK19 negative) and rest of the thyroid normal

\section{Conclusion}

De novo papillary carcinoma in a TGDC is rare. The diagnosis in most cases is incidental after surgical resection. Total thyroidectomy would be indicated, if the carcinomatous component is $>1 \mathrm{~cm}$ or $<1 \mathrm{~cm}$ but with enlarged cervical lymph nodes, tumour invasion of the cyst wall or an abnormal thyroid gland 